\begin{tabular}{|c|l|}
\hline Title & Facile Hydrothermal Preparation and Photocatalytic A ctivity of Bismuth Tungstate Polycrystall line Flake ball Particles \\
\hline Author(s) & A mano, Fumiaki; Nogami, Kohei; A be, Ryu; Ohtani, Bunsho \\
\hline Citation & $\begin{array}{l}\text { Chemistry Letters, 36(11), 1314.1315 } \\
\text { https://doi.org/10.1246/1.2007.1314 }\end{array}$ \\
\hline Issue Date & 2007 \\
\hline Doc URL & http://hdl.handle.net/2115/48667 \\
\hline Type & article(author version) \\
\hline File Information & ChemLett36_1314.pdf \\
\hline
\end{tabular}

Instructions for use 


\title{
Facile Hydrothermal Synthesis and Photocatalytic Activity of Bismuth Tungstate Polycrystalline Flake-ball Particles
}

\author{
Fumiaki Amano, ${ }^{1,2}$ Kohei Nogami, ${ }^{2}$ Ryu Abe, ${ }^{1,2}$ and Bunsho Ohtani*1,2 \\ ${ }^{1}$ Graduate School of Environmental Science, Hokkaido University, Sapporo 060-0810 \\ ${ }^{2}$ Catalysis Research Center, Hokkaido University, Sapporo 001-0021
}

(Received August 1, 2007; CL-070818; E-mail: ohtani@cat.hokudai.ac.jp)

Micrometer-sized spherical particles of bismuth tungstate $\left(\mathrm{Bi}_{2} \mathrm{WO}_{6}\right)$ with hierarchical architecture were prepared by facile hydrothermal reaction without using any surfactants and polymers as structure-directing agents. The particles were assemblies of polycrystalline flakes composed of rectangular platelets. The hierarchical polycrystalline particles of "flake-ball" shape exhibited relatively high photocatalytic activity for oxidative decomposition of acetic acid in aqueous suspensions.

Preparations of hierarchical architectures by assembling nanomaterials of low-dimensional morphologies as building blocks have attracted much attention because of their expected novel properties and functions depending on the size, shape, orientation, alignment, or dimensionality. ${ }^{1}$ Controlled solution-growth routes have often been used as bottom-up technology for anisotropic crystal growth of nanomaterials to construct hierarchical architectures. Recently, Huang et al. reported a hydrothermal synthesis of micrometer-sized particles of bismuth tungstate $\left(\mathrm{Bi}_{2} \mathrm{WO}_{6}\right)$ composed of a number of plate-like nanomaterials using poly(vinyl pyrrolidone) (PVP) as a structure-directing agent without showing the photocatalytic activity of the samples. ${ }^{2}$ It has been reported that $\mathrm{Bi}_{2} \mathrm{WO}_{6}$, which is a layered oxide and a member of cation-deficient Aurivillius phases, shows photocatalytic activity under visible-light irradiation for oxygen liberation from water and degradation of organic dye molecules in water. $^{3-7}$ We have developed, independently from the work by Huang's group, a facile hydrothermal reaction (HTR) of sodium tungstate $\left(\mathrm{Na}_{2} \mathrm{WO}_{4}\right)$ and bismuth nitrate $\left(\mathrm{Bi}\left(\mathrm{NO}_{3}\right)_{3}\right)$ at $433 \mathrm{~K}$ for the preparation of micrometersized $\mathrm{Bi}_{2} \mathrm{WO}_{6}$ polycrystalline particles with a hierarchical structure without using any structure-directing agents. The photocatalytic activities of the obtained hierarchicalstructured $\mathrm{Bi}_{2} \mathrm{WO}_{6}$ particles were studied for several types of photocatalytic reactions. The experimental details are given in Supporting Information on the web site.

$\mathrm{X}$-ray diffraction (XRD) patterns of $\mathrm{Bi}_{2} \mathrm{WO}_{6}$ prepared by HTR and conventional solid-state reaction (SSR) of bismuth oxide $\left(\mathrm{Bi}_{2} \mathrm{O}_{3}, 99.9 \%\right)$ and tungsten oxide $\left(\mathrm{WO}_{3}, 99.99 \%\right)$ at $1173 \mathrm{~K}$ are shown in Figure S1. ${ }^{8}$ The XRD patterns of both samples closely resemble that of reported data $\left(\mathrm{Bi}_{2} \mathrm{WO}_{6}\right.$, JCPDS Card number: 39-0256). The HTR sample gave peaks broader than those of the SSR sample, indicating smaller size of the HTR crystallites; crystallite sizes of $\mathrm{Bi}_{2} \mathrm{WO}_{6}$ calculated from FWHM of the most intense 131 diffraction peak at 28.8 deg. using Scherrer's equation were 17 and $350 \mathrm{~nm}$ for HTR and SSR samples, respectively.

Panels (a)-(c) in Figure 1 are images of the HTR sample taken by field-emission type scanning electron microscopy
(FE-SEM). Spherical particles with an average diameter of 3$4 \mu \mathrm{m}$ were observed in a low magnification image (Figure 1a). An enlarged view (Figure 1b) showed that each particle was of ball-like shape consisting of an assembly of flakes radiating in all directions ("flake-ball"). A high magnification image (Figure 1c) shows that the flakes are composed of rectangular platelets with lateral sizes of a few hundreds of nanometers and thicknesses of 20-35 $\mathrm{nm}$. On the other hand, the SSR samples seem to be accumulated plates of random shape (Figure 1d). Thus, the HTR particles have hierarchical structure: the primary structure is a layered $\mathrm{Bi}_{2} \mathrm{WO}_{6}$ crystal of alternate stacking of a $\left(\mathrm{Bi}_{2} \mathrm{O}_{2}\right)^{2+}$ layer and $\mathrm{WO}_{6}$ octahedron. The secondary structure is polycrystalline flakes of accumulated layered crystallites, which are assembled to flake-ball spherical particles as a tertiary structure. The hierarchical structure of the flake-ball particles resembles that of the recently reported $\mathrm{Bi}_{2} \mathrm{WO}_{6}$ prepared by PVP-assisted HTR, in which PVP might be an indispensable additive for the hierarchical structure. ${ }^{2}$ However, our method is simple; no organic additives are needed. Use of $10 \%$ excess feed of $\mathrm{Na}_{2} \mathrm{WO}_{4}$ and HTR under strongly acidic ( $\mathrm{pH} 1.2$ ) conditions induced the production of uniform flake-ball particles in relatively high yield, though the HTR conditions have not been optimized.

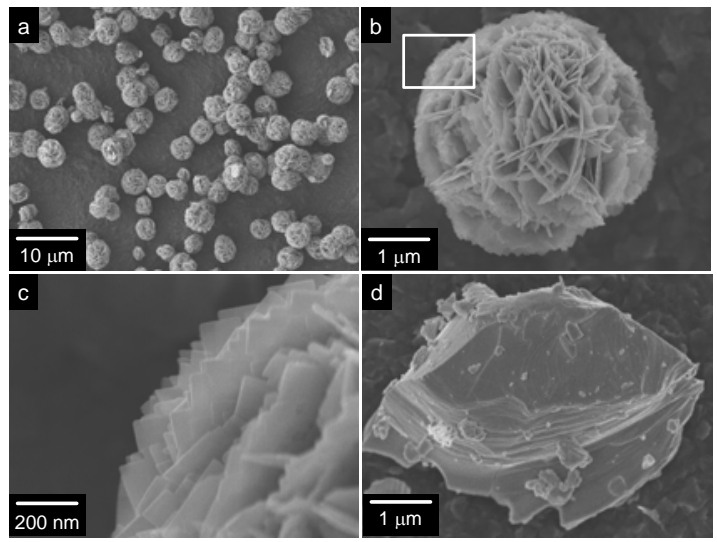

Figure 1. FE-SEM images of $\mathrm{Bi}_{2} \mathrm{WO}_{6}$ prepared by HTR (a)-(c) and SSR (d). The image in (c) is a high magnification image of a part of (b).

The mechanism of flake-ball particle formation was elucidated by XRD and FE-SEM analysis of samples collected during HTR. In the initial stage $(1 \mathrm{~h})$, amorphous nanoparticles were formed, and additional HTR $(2 \mathrm{~h})$ gave irregular-shaped (not spherical) small $(<2 \mu \mathrm{m})$ flake aggregates which gave an XRD pattern of a poorly crystallized $\mathrm{Bi}_{2} \mathrm{WO}_{6}$ phase. This suggests that flake-ball particles were produced through crystal-plane selective growth starting from cores, small amorphous or poorly 
crystallized polycrystalline particles of $\mathrm{Bi}_{2} \mathrm{WO}_{6}$, but not through aggregation of rectangular platelets of high crystallinity once produced independently. The crystal growth of flake aggregates probably proceeds through Ostwald ripening process, in which smaller particles disappear due to their high solubility and crystallize to larger particles again, and minimization of surface free energy might result in the formation of spherical particles. From FE-SEM observation, rectangular platelets appeared as parts of flakes by 5-h HTR. Crystallization of such plate-like $\mathrm{Bi}_{2} \mathrm{WO}_{6}$ crystallites was previously found using the HTR method in the absence of structure-directing agents. ${ }^{5,6}$ The XRD patterns of flake-ball particles were almost unchanged after 5-h HTR.

Photocatalytic activity of the polycrystalline flake-ball particles was investigated using several types of photocatalytic reactions. Figure 2 shows liberation of carbon dioxide $\left(\mathrm{CO}_{2}\right)$ during photocatalytic $(\lambda>300 \mathrm{~nm})$ decomposition of acetic acid in aerated aqueous suspensions. The flake-ball particles exhibited a much higher rate of $\mathrm{CO}_{2}$ liberation than that of the SSR sample. The rate by flake-ball particles $\left(20.3 \mu \mathrm{mol} \mathrm{h}{ }^{-1}\right)$ was comparable to that of anatase titanium(IV) oxide $\left(\mathrm{TiO}_{2}\right.$, Ishihara Sangyo ST-01, $21.0 \mu \mathrm{mol}$ $\mathrm{h}^{-1}$ ). The high activity might not be due to the $10 \%$ excess amount of tungsten, i.e., a plausible presence of amorphous $\mathrm{WO}_{3}$, since $\mathrm{WO}_{3}$ exhibited a very slow rate of $\mathrm{CO}_{2}$ liberation (Figure 2c). It should be noted that the flake-ball particles could induce oxidative decomposition of acetic acid even under visible light irradiation $(\lambda>400 \mathrm{~nm})$ by eliminating ultraviolet light using a cut-off filter (Kenko L42) with a relatively slow rate $\left(1.96 \mu \mathrm{mol} \mathrm{h}{ }^{-1}\right)$ compared with that under irradiation without using the filter. The band gap energy of the flake-ball particles, which was estimated from the diffuse reflectance photo-absorption spectrum assuming indirect allowed transition, was $2.9 \mathrm{eV}$, indicating absorption of visible light at ca. $400-430 \mathrm{~nm}$. In the case of ST-01, decomposition of acetic acid was negligible under visible light irradiation $\left(\mathrm{CO}_{2}\right.$ liberation rate: $\left.0.25 \mu \mathrm{mol} \mathrm{h}^{-1}\right)$.

Zhu et al. reported that $\mathrm{Bi}_{2} \mathrm{WO}_{6}$ was unstable and transformed to tungstic acid $\left(\mathrm{H}_{2} \mathrm{WO}_{4}\right)$ and $\mathrm{Bi}_{2} \mathrm{O}_{3}$ in acidic solutions ( $\mathrm{pH}$ 4.7). ${ }^{6}$ However, in our studies, XRD measurement revealed that the crystal structure was not changed in $5 \mathrm{vol} \%$ acetic acid solution. It was also confirmed that the hierarchical structure was maintained during the photocatalytic reaction as revealed by FE-SEM observation.

The flake-ball particles exhibited photocatalytic activity higher than that of the SSR sample also in other photocatalytic reactions, such as oxidative decomposition of gaseous acetaldehyde in air and oxygen liberation from water in the presence of silver sulfate as a sacrificial electron acceptor (Table 1). The BET specific surface area of the flake-ball particles is much larger than that of the SSR sample. The large specific surface area may enhance adsorption of substrates and decrease diffusion length of photogenerated carriers. In addition to this surface-area effect, the high crystallinity of $\mathrm{Bi}_{2} \mathrm{WO}_{6}$, which was proved by well-developed crystal shape of rectangular platelets in the flake-ball particles in FE-SEM analyses, must improve the photocatalytic activity. It has been suggested that high crystallinity is one of requisites for high photocatalytic activity to reduce probability in recombination of photogenerated carriers at crystalline defects. The flake-ball structure enables to combine large surface area and high crystallinity at the same time, while for simple primary particles poorly crystallized small particles or highly crystallized large particles are generally obtained. Although further study is needed on the effect of anisotropic morphology of the rectangular platelet, which exhibited a developed large crystal plane, on the photocatalytic activity, superior photocatalytic activity of flake-ball particles owing to their large surface area and high crystallinity was proved.

It should be noted that the flake-ball particles are readily separated from a solution by sedimentation due to the micrometer-sized macro structure. Recovery of the powders from water seems to be a critical issue when ultra-fine photocatalyst powders are used in aqueous systems. ${ }^{9}$ The $\mathrm{Bi}_{2} \mathrm{WO}_{6}$ flake-ball particles were precipitated from an aqueous suspension in less than $1 \mathrm{~h}$, while a suspension of the ultrafine powders of $\mathrm{WO}_{3}$ was unclear after $1 \mathrm{~h}$ (Figure S2). ${ }^{8}$

It is concluded that controlling the hierarchical architectures of photocatalyst crystalline assemblies is beneficial for the preparation of highly active photocatalysts for oxidative decomposition of organic pollutants and for practical use in water purification systems because of the feasible separation by sedimentation and filtration.

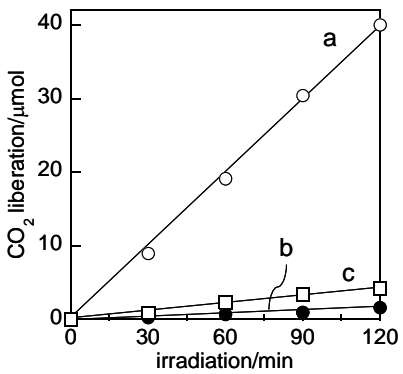

Figure 2. Photocatalytic oxidative decomposition of acetic acid over $\mathrm{Bi}_{2} \mathrm{WO}_{6}$ prepared by (a) HTR and (b) SSR and $\mathrm{WO}_{3}$ (c). For details, see $\mathrm{SI}^{8}$

Table 1. Specific surface area and photocatalytic activities of $\mathrm{Bi}_{2} \mathrm{WO}_{6}$ prepared by HTR and SSR.

\begin{tabular}{cccc}
\hline sample & $S_{\mathrm{BET}}{ }^{\mathrm{a}} / \mathrm{m}^{2} \mathrm{~g}^{-1}$ & $R\left(\mathrm{CO}_{2}\right)^{\mathrm{b}} / \mu \mathrm{mol} \mathrm{h}^{-1}$ & $\mathrm{O}_{2}{ }^{\mathrm{c}} / \mu \mathrm{mol}$ \\
\hline HTR & 21.2 & 43.6 & 1.83 \\
SSR & 0.2 & 10.9 & 0.22 \\
\hline
\end{tabular}

${ }^{\mathrm{a} B E T}$ specific surface area measured by nitrogen adsorption at $77 \mathrm{~K}$. ${ }^{b}$ Initial rate of $\mathrm{CO}_{2}$ liberation in the photocatalytic decomposition of acetaldehyde in air. For details, see SI. ${ }^{8}$

${ }^{\mathrm{c}}$ Amount of oxygen liberated from aqueous solution of silver sulfate by 30-min photoirradiation. For details, see SI. ${ }^{8}$

\section{References and Notes}

1 H. T. Shi, L. M. Qi, J. M. Ma, H. M. Cheng, J. Am. Chem. Soc. 2003, 125, 3450.

2 Y. Y. Li, J. P. Liu, X. T. Huang, G. G. Li, Cryst. Growth Des. 2007, 7, 1350 .

3 A. Kudo, S. Hijii, Chem. Lett. 1999, 1103.

4 J. W. Tang, Z. G. Zou, J. H. Ye, Catal. Lett. 2004, 92, 53.

5 C. Zhang, Y. F. Zhu, Chem. Mater. 2005, 17, 3537.

6 H. B. Fu, C. S. Pan, W. Q. Yao, Y. F. Zhu, J. Phys. Chem. B 2005, 109, 22432

7 J. G. Yu, J. F. Xiong, B. Cheng, Y. Yu, J. B. Wang, J. Solid State Chem. 2005, 178, 1968.

8 Supporting Information is available electronically on the CSJJournal Web site, http://www.csj.jp/journals/chemlett/index.html.

9 H. Y. Zhu, Y. Lan, X. P. Gao, S. P. Ringer, Z. F. Zheng, D. Y. Song, J. C. Zhao, J. Am. Chem. Soc. 2005, 127, 6730. 